\title{
DNA 5-hydroxymethylcytosine in pediatric central nervous system tumors may impact tumor classification and is a positive prognostic marker
}

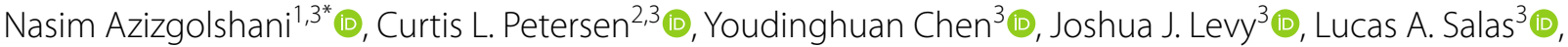 \\ Laurent Perreard ${ }^{3}$, Lananh N. Nguyen ${ }^{4,5}$ and Brock C. Christensen ${ }^{1,3}$ (i)
}

\begin{abstract}
Background: Nucleotide-specific 5-hydroxymethylcytosine $(5 \mathrm{hmC})$ remains understudied in pediatric central nervous system (CNS) tumors. $5 \mathrm{hmC}$ is abundant in the brain, and alterations to $5 \mathrm{hmC}$ in adult CNS tumors have been reported. However, traditional approaches to measure DNA methylation do not distinguish between 5-methylcytosine $(5 \mathrm{mC})$ and its oxidized counterpart $5 \mathrm{hmC}$, including those used to build CNS tumor DNA methylation classification systems. We measured $5 \mathrm{hmC}$ and $5 \mathrm{mC}$ epigenome-wide at nucleotide resolution in glioma, ependymoma, and embryonal tumors from children, as well as control pediatric brain tissues using tandem bisulfite and oxidative bisulfite treatments followed by hybridization to the Illumina Methylation EPIC Array that interrogates over 860,000 CpG loci.
\end{abstract}

Results: Linear mixed effects models adjusted for age and sex tested the CpG-specific differences in $5 \mathrm{hmC}$ between tumor and non-tumor samples, as well as between tumor subtypes. Results from model-based clustering of tumors was used to test the relation of cluster membership with patient survival through multivariable Cox proportional hazards regression. We also assessed the robustness of multiple epigenetic CNS tumor classification methods to $5 \mathrm{mC}$ specific data in both pediatric and adult CNS tumors. Compared to non-tumor samples, tumors were hypohydroxymethylated across the epigenome and tumor $5 \mathrm{hmC}$ localized to regulatory elements crucial to cell identity, including transcription factor binding sites and super-enhancers. Differentially hydroxymethylated loci among tumor subtypes tended to be hypermethylated and disproportionally found in CTCF binding sites and genes related to posttranscriptional RNA regulation, such as DICER1. Model-based clustering results indicated that patients with low $5 \mathrm{hmC}$ patterns have poorer overall survival and increased risk of recurrence. Our results suggest $5 \mathrm{mC}$-specific data from OxBS-treated samples impacts methylation-based tumor classification systems giving new opportunities for further refinement of classifiers for both pediatric and adult tumors.

Conclusions: We identified that $5 \mathrm{hmC}$ localizes to super-enhancers, and genes commonly implicated in pediatric CNS tumors were differentially hypohydroxymethylated. We demonstrated that distinguishing methylation and hydroxymethylation is critical in identifying tumor-related epigenetic changes. These results have implications for

*Correspondence: nasimgolshn@gmail.com

${ }^{1}$ Department of Molecular and Systems Biology, Geisel School

of Medicine at Dartmouth, Lebanon, NH 03756, USA

Full list of author information is available at the end of the article

(C) The Author(s) 2021. Open Access This article is licensed under a Creative Commons Attribution 4.0 International License, which permits use, sharing, adaptation, distribution and reproduction in any medium or format, as long as you give appropriate credit to the original author(s) and the source, provide a link to the Creative Commons licence, and indicate if changes were made. The images or other third party material in this article are included in the article's Creative Commons licence, unless indicated otherwise in a credit line to the material. If material is not included in the article's Creative Commons licence and your intended use is not permitted by statutory regulation or exceeds the permitted use, you will need to obtain permission directly from the copyright holder. To view a copy of this licence, visit http://creativecommons.org/licenses/by/4.0/. The Creative Commons Public Domain Dedication waiver (http://creativeco mmons.org/publicdomain/zero/1.0/) applies to the data made available in this article, unless otherwise stated in a credit line to the data. 
patient prognostication, considerations of epigenetic therapy in CNS tumors, and for emerging molecular neuropathology classification approaches.

Keywords: Epigenetics, 5-Hydroxymethylcytosine, Pediatric CNS/brain tumors, Cancer, Methylation

\section{Background}

Central nervous system malignancies are the most common pediatric solid tumor type in North America [1]. They are the leading cause of death from disease in childhood and are the greatest source of cancer-derived morbidity in the USA, due to treatment side effects and resistance [2]. Pathologists commonly classify these tumors into glioma, ependymoma, and embryonal types [3]. Within these broad groups, there is substantial variability in histopathology and patient prognosis. For instance, gliomas encompass diagnoses such as glioblastoma, a high-grade cancer with low survival requiring chemotherapy, radiation, and surgery, while pilocytic astrocytoma, a benign entity, is cured by surgery alone. Due to the variation in underlying disease mechanism and impact on prognosis, risk stratification and classification are critical to treatment planning and goal setting.

Several studies have paved the way in using molecular markers to classify pediatric central nervous system (CNS) tumors. Through gene sequencing, diagnoses have been subdivided. WNT1 and $S H H$ mutations have been identified as subtype-specific genetic alterations in group 1 and 2 medulloblastomas with subsequent treatment and prognostic implications [4, 5] Likewise, ependymomas have been split into multiple subgroups based on C11orf95-RELA and Yap1 fusions [6], while BRAFKIAA1549 fusion has been used to characterize pediatric low-grade gliomas [7]. These molecular markers have recently been incorporated into the WHO CNS tumor classification guidelines [3]. However, while genomic approaches have provided ways to better characterize pediatric CNS tumors, many lack obvious genetic drivers. Further, these genetic findings are not always associated with prognosis.

While genome-scale $5 \mathrm{mC}$ profiles have been welldescribed and used clinically in CNS tumors, there is very limited nucleotide-specific data for the other major cytosine modification in the brain, 5-hydroxymethylcytosine $(5 \mathrm{hmC})$. In mammals, TET proteins oxidize $5 \mathrm{mC}$ to $5 \mathrm{hmC}$, which can be further transformed to 5 -formylcytosine $(5 \mathrm{fC})$ and 5 -carboxylcytosine $(5 \mathrm{caC})$ leading to methylation loss $[8,9]$. Though initially thought to be an intermediate, $5 \mathrm{hmC}$ has since been shown to play a distinct role in regulating gene transcription and increasing chromatin accessibility [10-12]. Unlike methylcytosine, $5 \mathrm{hmC}$ concentrates in gene bodies and enhancers and is associated with increased gene expression [13-15]. $5 \mathrm{hmC}$ tends to be depleted in promoter $\mathrm{CpG}$ islands and is instead observed in the flanking $\mathrm{CpG}$ shores and shelves $[14,16]$.

$5 \mathrm{hmC}$ modifications are most abundant in the brain, with levels tenfold higher than other tissues, though varying among regions of the brain [17]. In the cerebellum, $5 \mathrm{hmC}$ accounts for up to $42 \%$ of cytosine modifications [18]. Appropriate patterning of $5 \mathrm{hmC}$ is likely critical to normal neurodevelopment, and altered $5 \mathrm{hmC}$ has been associated with developmental neuropathologies such as Rett syndrome, autism, and schizophrenia [19-21]. $5 \mathrm{hmC}$ has been studied in adult brain tumors, such as glioblastoma, and has been shown to correlate with survival $[13,22,23]$. However, genomewide nucleotide level studies in pediatric central nervous system tumors are lacking. Changes in $5 \mathrm{hmC}$ in tumors have been consistent across tissue types, with depletion of $5 \mathrm{hmC}$ in gene bodies and regulatory regions such as enhancers and transcription factor binding sites [24-28]. Loss of $5 \mathrm{hmC}$ tends to coincide with commonly mutated genes in cancers and is tissuespecific $[10,13,29,30] .5 \mathrm{hmC}$ appears to play a unique role in cancer, such as localizing to sites of DNA damage, and promoters with $5 \mathrm{hmC}$ have been shown to be resistant to the hypermethylation that is common in cancers $[28,31]$. To date, the role of $5 \mathrm{hmC}$ in pediatric CNS tumors has yet to be adequately measured and understood.

Epigenetic profiling to characterize CNS tumors in both research and clinical settings is increasingly applied. DNA methylation, the addition of a methyl group to cytosine, 5 -methylcytosine $(5 \mathrm{mC})$, is one of the most common and best-studied epigenetic modifications. Alterations in the methylation status of specific cytosines have been used to classify CNS tumors in both adults and children to great effect-identifying changes that transcend somatic genetic alterations [32-36]. Capper et al. have even set up a molecular neuropathology webite where methylation array data can be uploaded to classify CNS tumors [36]. Tumor DNA methylation signatures have been used not only for diagnostic purposes but to stratify subgroups with a worse prognosis [37, 38]. However, current methods of measuring nucleotide level $5 \mathrm{mC}$-including those for CNS tumor classification-rely on bisulfite conversion techniques which cannot distinguish between $5 \mathrm{hmC}$ and $5 \mathrm{mC}$ [39]. This confounds to what degree these 
classifications rely on $5 \mathrm{hmC}$ to diagnose or prognosticate tumor types and has not been clearly delineated.

Most studies of $5 \mathrm{hmC}$ only provide a genome-level summary measure of $5 \mathrm{hmC}$ without chromosome, gene region, or nucleotide-specific information [40, 41]. Due to the abundance of $5 \mathrm{hmC}$ in the brain and its observed alterations in tumorigenesis, we aimed to examine $5 \mathrm{hmC}$ in pediatric $\mathrm{CNS}$ tumors. We measured 5-methylcytosine and 5-hydroxymethylcytosine epigenome-wide at nucleotide resolution in pediatric glioma, ependymoma, and embryonal tumors as well as non-tumor tissue using tandem bisulfite and oxidative bisulfite sequencing followed by hybridization to the Illumina Methylation EPIC Array that interrogates over $860,000 \mathrm{CpG}$ loci. Lastly, we used our raw data, processed with bisulfite treatment (including both $5 \mathrm{mC}$ and $5 \mathrm{hmC}$ ) as well as oxidative bisulfitetreated data files (including only $5 \mathrm{mC}$ ), as the input for several existing tumor classification systems to determine whether using $5 \mathrm{mC}$-specific data could impact the diagnostic aptitude of these tools.

\section{Results}

\section{Overview of study population}

All cases included in this study $(n=27)$ received treatment at the Children's Hospital at Dartmouth Hitchcock and the Norris Cotton Cancer Center between 1993 and 2009. The mean age of cases was 9.5 years, and the mean age of subjects providing non-tumor samples was 6 years. Tumor samples included thirteen gliomas, eight ependymomas, and six embryonal tumors. Additional details of subject demographic, tumor characteristics and diagnoses are provided in Table 1. Our cohort included both common and rare entities, including dysplastic gangliocytoma and spinal myxopapillary ependymoma. The most common glioma subtype was pilocytic astrocytoma, and medulloblastoma for embryonal tumors. The most diverse subtype was gliomas with a range of histopathologic diagnoses incluging desmoplastic ganglioglioma, dysembryoplastic neuropithelial tumors, and dysplastic gangliocytomas with limited sample sizes due to their respective rare prevalence (Table 1 ). All samples were H3F3A (K27 and G34) and TERT promoter (C228T and $\mathrm{C} 250 \mathrm{~T}$ ) wild-type, as determined by Sanger sequencing (Additional file 6: Table S1).

\section{Pediatric CNS tumors are globally depleted of $5 \mathrm{hmC}$}

To measure nucleotide-specific $5 \mathrm{hmC}$ and $5 \mathrm{mC}$ levels, we applied tandem bisulfite and oxidative bisulfite treatment to DNA from 27 fresh frozen pediatric CNS tumor samples and three non-tumor control tissues. Treated DNA was hybridized to the Illumina Human Methylation EPIC array. Following processing, CpG probes associated
Table 1 Patient demographics and tumor characteristics

\begin{tabular}{|c|c|c|}
\hline & Tumor $(n=27)$ & Non-Tumor $(n=3)$ \\
\hline Age, mean (SD) & $9.5(5.5)$ & $6(5.6)$ \\
\hline Range & $1-18$ & $0-11$ \\
\hline \multicolumn{3}{|l|}{$\operatorname{sexn}(\%)$} \\
\hline Female & $12(44)$ & $1(33)$ \\
\hline Male & $15(56)$ & $2(67)$ \\
\hline \multicolumn{3}{|l|}{ Tumor type, ${ }^{\mathrm{a}} n(\%)$} \\
\hline Embryonal & $6(22)$ & \\
\hline Medulloblastoma & 4 & \\
\hline Embryonal (NOS) & 2 & \\
\hline Glioma & $13(48)$ & \\
\hline Anaplastic ganglioglioma & 1 & \\
\hline Desmoplastic ganglioglioma & 2 & \\
\hline $\begin{array}{l}\text { Dysembryoplastic neuroepithelial } \\
\text { tumor }\end{array}$ & 2 & \\
\hline Dysplastic gangliocytoma & 1 & \\
\hline Glioblastoma & 1 & \\
\hline Pilocytic astrocytoma & 5 & \\
\hline Low-grade glioma & 1 & \\
\hline Ependymoma & $7(30)$ & \\
\hline Subependymoma & 1 & \\
\hline Ependymoma & 5 & \\
\hline Myxopapillary ependymoma & 1 & \\
\hline \multicolumn{3}{|l|}{ WHO grade $n(\%)$} \\
\hline I & $12(44)$ & \\
\hline$\|$ & $5(19)$ & \\
\hline III & $2(7)$ & \\
\hline IV & $8(30)$ & \\
\hline \multicolumn{3}{|l|}{ Sample location n (\%) } \\
\hline Supratentorial & $9(33)$ & $3(100)$ \\
\hline Subtentorial & $17(63)$ & \\
\hline Spinal & $1(4)$ & \\
\hline Median follow up (years) & 14 & \\
\hline Mean time to recurrence (years) & 1.5 & \\
\hline
\end{tabular}

${ }^{a}$ Tumor type and grade were determined by a neuropathologist re-review of samples and distribution of tumor type was determined by prevalence at the institution

with SNPs and sex chromosomes were removed, and 743,461 CpG sites remained in the dataset.

To first compare our findings with prior work where $5 \mathrm{hmC}$ measures only provide a single epigenome-wide summary value, we calculated median $5 \mathrm{hmC}$ levels using the 743,461 CpG sites in our dataset. Median $5 \mathrm{hmC}$ levels were significantly lower in tumors (gliomas $1.75 \%$, ependymomas $1.76 \%$, and embryonal tumors $1.22 \%$ ), compared to non-tumor tissues (4.81\%) (Fig. 1a). Median levels of tumor $5 \mathrm{mC}$ (glioma $62.5 \%$, ependymoma $62.4 \%$, embryonal $57.7 \%$, non-tumor $60.3 \%$ ), did not differ from controls (Fig. 1b). 


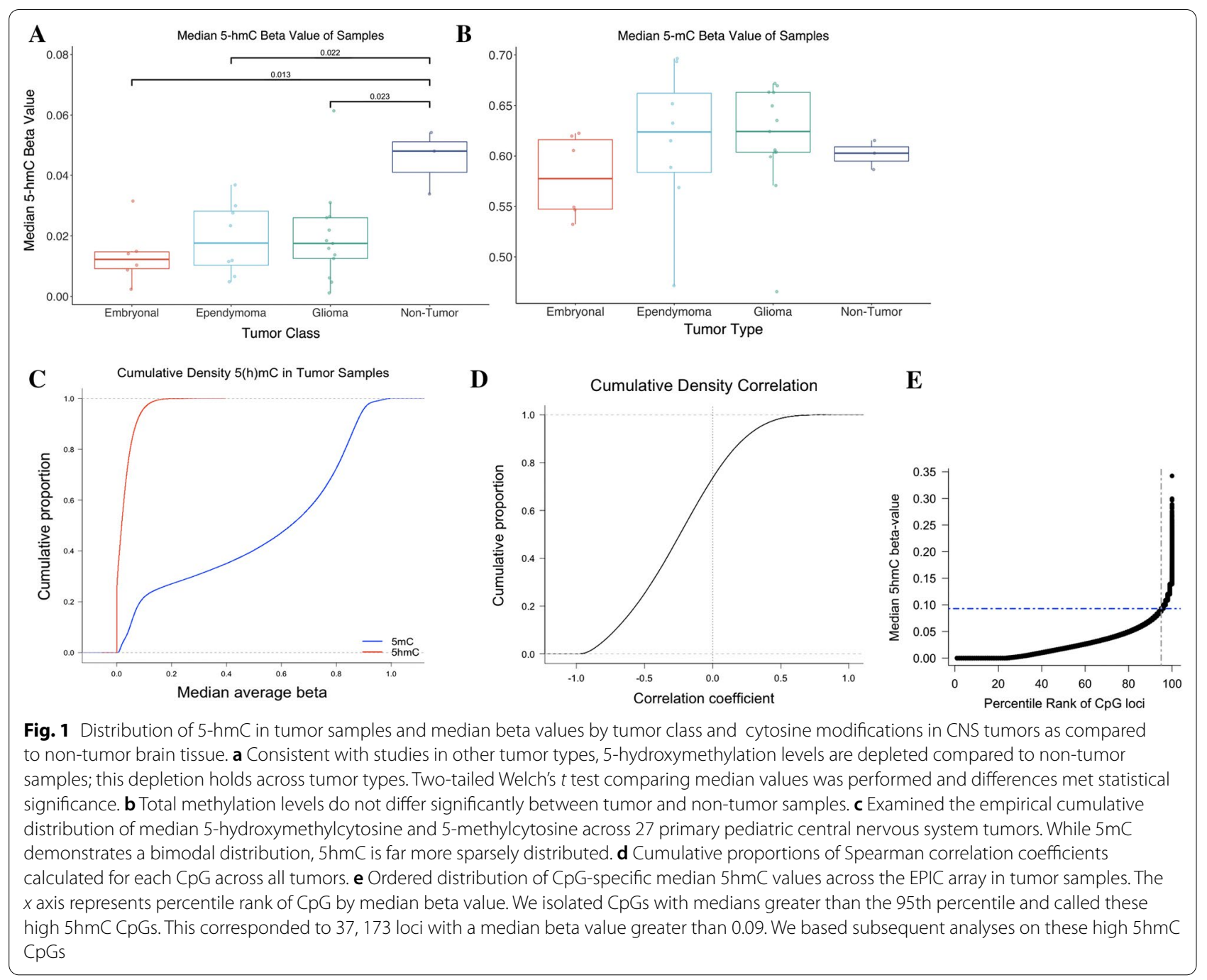

Cumulative density plots for median $5 \mathrm{hmC}$ and $5 \mathrm{mC}$ across all tumor samples demonstrated very low $5 \mathrm{hmC}$ levels at the majority of $\mathrm{CpG}$ sites in contrast to $5 \mathrm{mC}$ levels, which were more evenly distributed (Fig. 1c). We observed a negative correlation between $5 \mathrm{hmC}$ and $5 \mathrm{mC}$ for approximately $80 \%$ of CpGs in tumor samples as indicated by the distribution of Spearman's correlation coefficients (Fig. 1d). CpG-specific median beta values were calculated across all tumor samples and ordered by increasing value to identify the distribution of $5 \mathrm{hmC}$ level and loci with appreciable 5-hydroxymethylation (Fig. 1e).

The difference in the levels of $5 \mathrm{hmC}$ and $5 \mathrm{mC}$ between tumors and controls depends on the distance from the transcription start site (TSS). Immediately surrounding the TSS, there was no discernible difference between tumors and non-tumor tissues, and levels of both cytosine modifications drop to near zero. At approximately 300 base pairs in the $5^{\prime}$ and 700 base pairs $3^{\prime}$ direction, tumors demonstrate hypohydroxymethylation and hypermethylation (Fig. 2). Compared with non-tumor control tissue, we observed consistently lower levels of $5 \mathrm{hmC}$ within each tumor subtype (Additional file 6: Fig. S1a). Despite lower median $5 \mathrm{hmC}$ near TSSs, a substantial proportion of high $5 \mathrm{hmC}$ CpGs are found within $5 \mathrm{~kb}$ of the TSS, with $14 \%$ of loci within 2000 base pairs upstream of the start site (Additional file 6: Fig. S1b).

We decided to focus on the top $5 \%$ of measured loci with the highest median $5 \mathrm{hmC}$ beta values, which we refer to as the "high $5 \mathrm{hmC}$ CpGs," for downstream investigation. This subset consisted of 37,173 loci with a median beta value greater than 0.09 (Fig. 1d). $5 \mathrm{mC}$ levels at these sites had similar relationships between tumors and non-tumors as at the genome wide level with tumor median levels significantly higher than nontumor (Additional file 6: Fig. S2). 


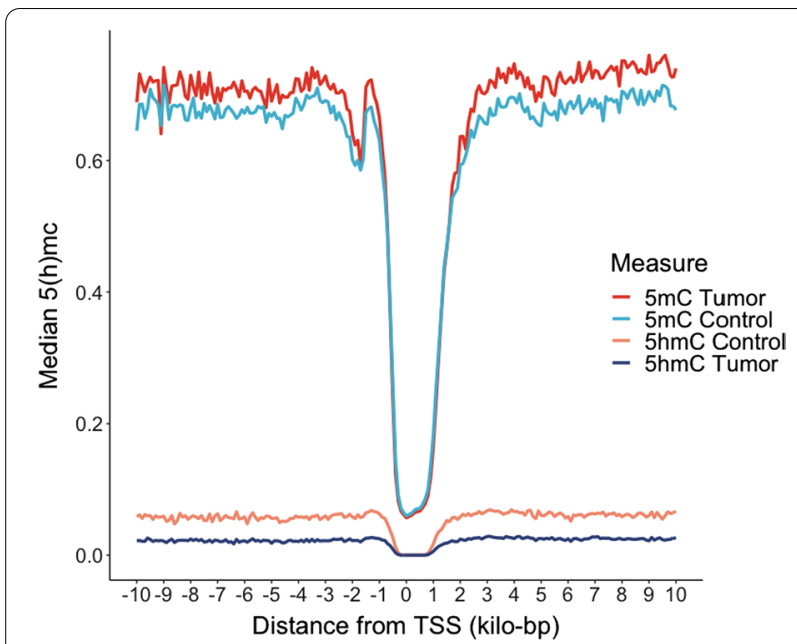

Fig. $25 \mathrm{hmC}$ levels in relation to transcription start sites. Median $5 \mathrm{hmC}$ and $5 \mathrm{mC}$ within \pm 10 kilo base pairs from the nearest gene transcription start site (TSS) for both tumors $(n=27)$ and non-tumors $(n=3)$. We observed that there were minimal differences between tumors and non-tumors near the transcription start sites and both cytosine modifications were depleted. However, within less than 1000 base pairs of the TSS, tumors were hypohydroxymethylated and hypermethylated as compared to controls

\section{Genomic enrichment of $5 \mathrm{hmC}$ in regulatory regions of the genome}

We then investigated the relationship between genomic context and CpGs with high $5 \mathrm{hmC}$, specifically investigating $\mathrm{CpG}$ islands and other genomic transcriptional regulatory elements (Fig. 3). Tumor $\mathrm{CpG}$ islands are significantly depleted of high 5hmC CpGs (OR 0.03, 95\% CI $0.03-0.03, P<2.2 \mathrm{E}-16)$. Out of the 37,173 loci designated as high $5 \mathrm{hmC}$, only 281 were identified as residing in the $\mathrm{CpG}$ islands. $\mathrm{CpG}$ depleted open sea regions are significantly enriched for high $5 \mathrm{hmC}$ CpGs (OR 2.71, 95\% CI $2.64-2.78, P<2.2 \mathrm{E}-16)$. In fact, $77 \%$ of high $5 \mathrm{hmC}$ loci were in open sea regions (Additional file 6: Table S2). High $5 \mathrm{hmC}$ sites predominantly localize to genomic regions associated with transcriptional regulation such as enhancers (OR 1.56, 95\% CI 1.49-1.64, $P=7.68 \mathrm{E}-69$ ), transcription factor binding sites (OR 1.14, 95\% CI 1.11$1.17, P=3.57 \mathrm{E}-20$ ), and $5^{\prime}$ untranslated regions (UTR-5) (OR 1.29, 95\% CI 1.25-1.33, $P=2.06 \mathrm{E}-61$ ).

Super-enhancers have been shown to be critical to cell identity, and aberrant methylation in these regions has been implicated in the development of human cancer [42]. To test the relation of super-enhancer with high $5 \mathrm{hmC}$ loci, we accessed super-enhancer coordinates defined in multiple brain-derived cell lines from the dbSuper database [43] and performed the same enrichment analysis as above. We identified significant enrichment of high $5 \mathrm{hmC}$ in annotated super-enhancers, including astrocyte lines (OR 1.75, 95\% CI 1.65-1.86, $P=1.94 \mathrm{E}-68)$ and anterior caudate lines (OR 1.93, 95\% CI 1.88-2.03, $P=6.22 \mathrm{E}-216$, Fig. 3).

Next, we investigated the enrichment of high $5 \mathrm{hmC}$ loci at the gene level. We wanted to identify genes that had large clusters of high $5 \mathrm{hmC}$ loci. We first identified genes with at least ten high $5 \mathrm{hmC} \mathrm{CpGs} \mathrm{(} n=486$ genes). We defined the gene-level high $5 \mathrm{hmC}$ sites as a proportion of measured CpGs that were high $5 \mathrm{hmC}$ to account for different gene lengths, number of $\mathrm{CpGs}$ measured on the array, and number of high $5 \mathrm{hmC}$ CpGs. The range of high $5 \mathrm{hmC}$ sites as a proportion of those measured ranged from 1.4 to $52.6 \%$ and the mean proportion of hydroxymethylated CpGs among the 486 genes was $17 \%$. Genes with the highest proportion of their CpGs designated as "high $5 \mathrm{hmC}$ " were SHOC2 (53\%), ZRANB1 (48\%), MBNL1 (41\%), ZMIZ1-AS1 (35\%), DICER1 (35\%), and GNAQ (35\%) (Additional file 2). This list also included, but was not exclusive to, genes pertaining to RNA interference pathways such as DICER1 and Argonaute gene (AGO2) [44-46] and neurodevelopment (GNAQ, FOXO3) [47, 48]. Using the genomic coordinates of the list of genes enriched for high $5 \mathrm{hmC}$ loci as the input, the Genomic Regions Enrichment of Annotations Tool (GREAT) analysis revealed biological pathways including regulation RNA interference pathways and RISC complex formation, as well as cell signaling pathways, and neuronal signaling and development. High $5 \mathrm{hmC}$ pathways also included those associated with craniofacial and neurodevelopmental pathologies, including autism, hyperactivity, and slanted palpebral fissures (Additional file 2).

\section{Locus-specific differentially hydroxymethylated regions}

We tested the high $5 \mathrm{hmC}$ sites for differential hydroxymethylation between tumor and non-tumors $(n=37,173)$, adjusting for age and sex, in a linear model. Tumors were hypohydroxymethylated, with $84 \%$ of the high $5 \mathrm{hmC}$ loci demonstrating reduced hydroxymethylation, and we observed 726 loci with significantly hypohydroxymethylation in tumors (FDR $<0.1$, Fig. 4a). Submitting the 726 differentially hypohydroxymethylated regions (DHMRs) to GREAT analysis resulted in overlap with steroid response pathways (4 of the 20 gene ontology biological processes), RNA stability, as well as WNT signaling and beta-catenin binding (Additional file 3). Furthermore, in contrast to the overall genomic context patterning of high $5 \mathrm{hmC}$ CpGs, tumor DHMRs were significantly enriched in $\mathrm{CpG}$ islands and shore regions while depleted in open sea regions (Fig. 4b). The majority of CpGs, 63\%, were found in open sea regions (Additional file 6: Table S3). Differentially hydroxymethylated regions (DHMRs) in CpG islands were associated with 


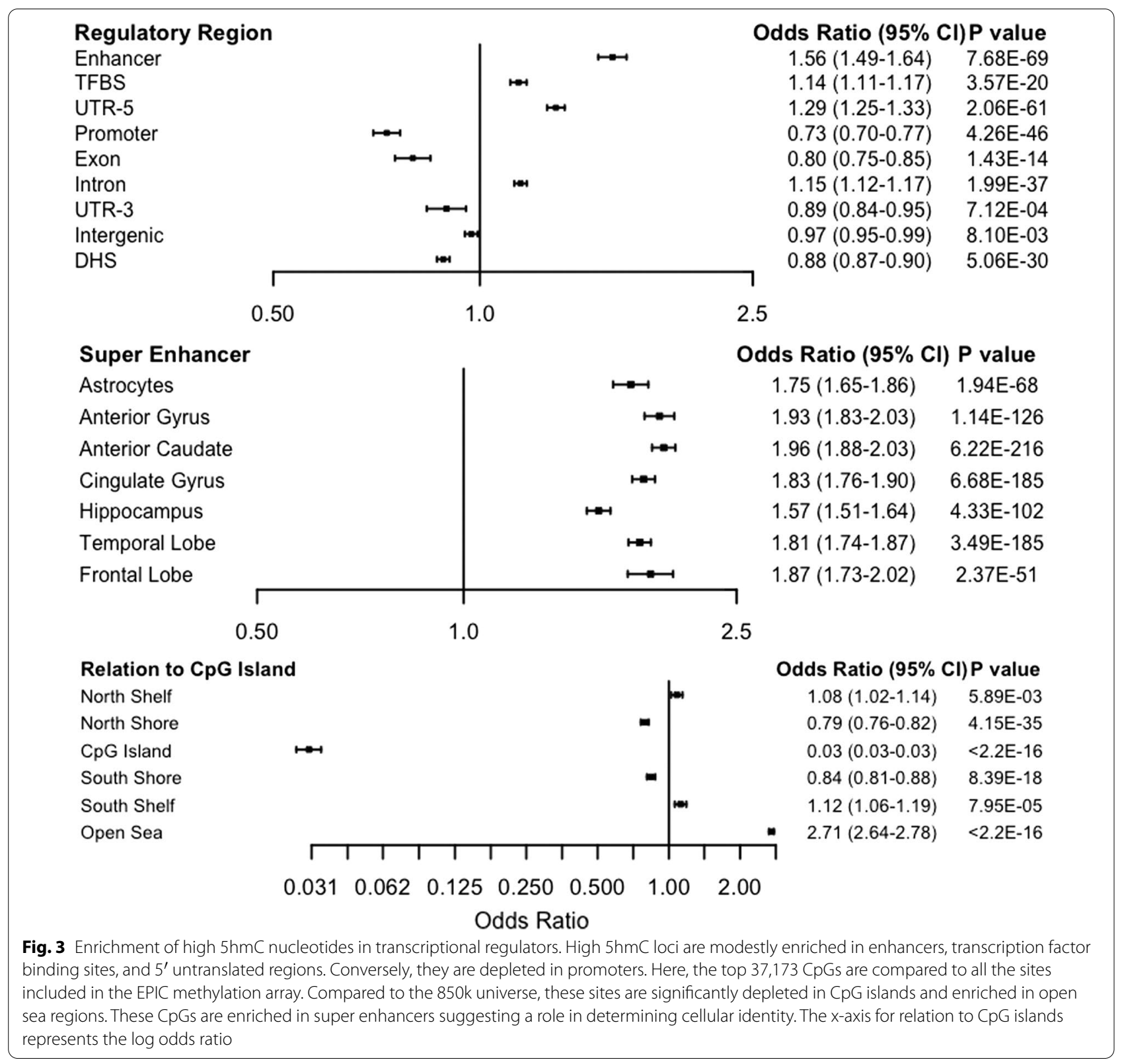

10 genes which included Wnt pathway regulator $A P C 2$, histone $\mathrm{H} 3$ demethylase $K D M 2 A$, and orphan G proteincoupled receptor GPRC5B critical for neurodevelopment (Additional file 3) [49].

To determine whether particular transcription factors are associated with DHMRs, we tested for enrichment in binding sites from transcription factor experiments (ENCODE) using the locus overlap analysis (LOLA) software in CNS and embryonic stem cell lines. We identified enrichment for DHMRs at CTCF binding sites, a transcription factor linked to alternative splicing by regulating RNA polymerase II and TET [50]
(Fig. 4d). Using LOLA to interrogate sites associated with histone modifications generated by the NIH Roadmap Epigenomics Project, we found that DHMRs were significantly associated both with chromatin signatures for transcriptional activation (H3K4me3) and priming (H3K4me1) (Additional file 6: Fig. S3). Lastly, we investigated total $5 \mathrm{mC}$ levels among DHMRs. All tumor subtypes were hypermethylated at these sites as compared to non-tumors (Fig. 4c). This pattern of hypohydroxymethylation and hypermethylation was only captured in tandem OxBS derived data and was lost in BS only 


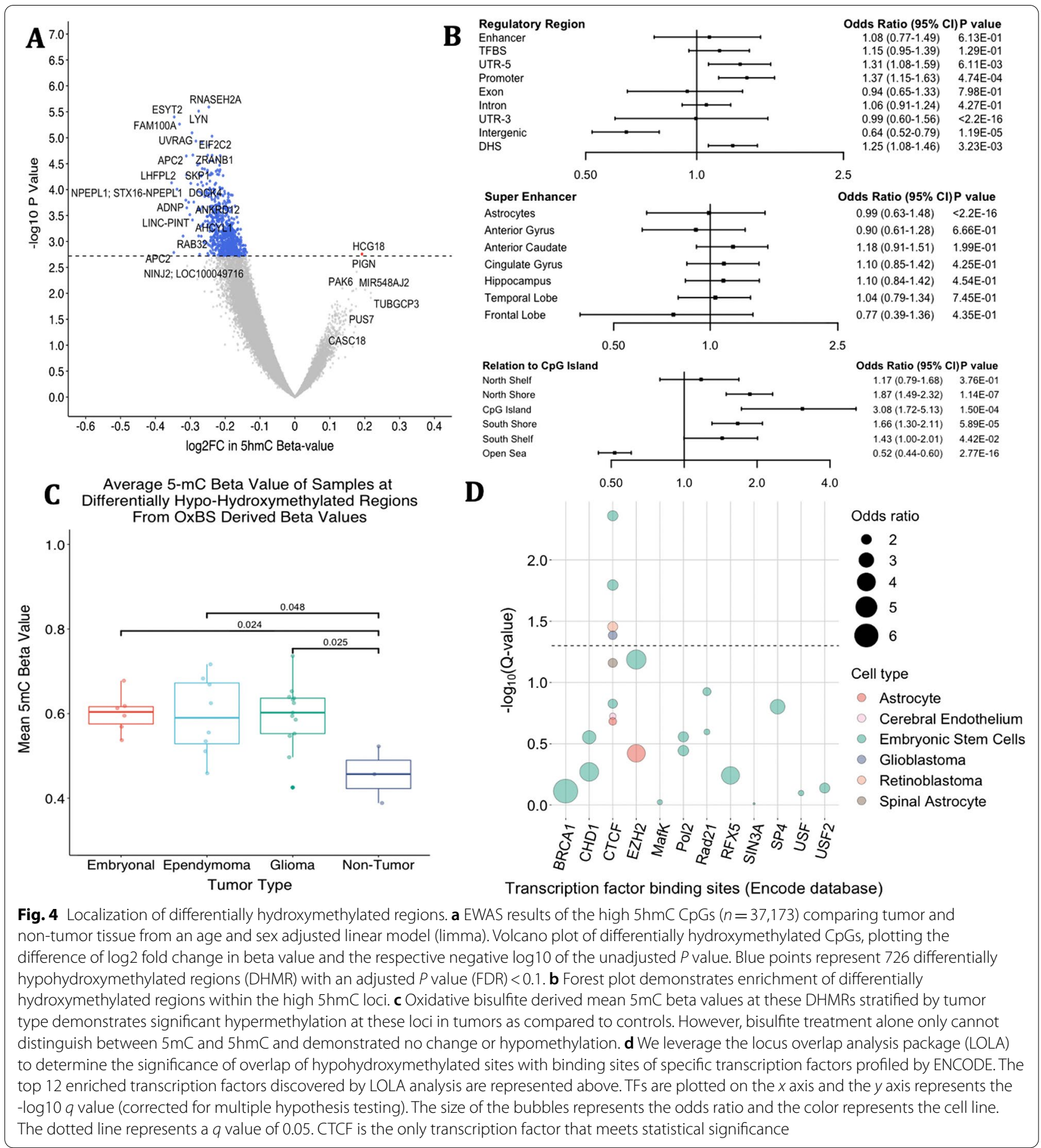

derived data with the latter demonstrating a relative hypomethylation in tumors (Additional file 6: Fig. S4). In tumors, these sites also demonstrated higher levels of $5 \mathrm{mC}$ compared to average $5 \mathrm{mC}$ levels in the rest of the genome (Additional file 6: Fig. S5).

\section{Subtype to non-tumor comparisons}

To further explore high 5 -hmC sites $(n=37,173)$ in tumor and non-tumor tissues, we stratified by tumor type (embryonal, ependymoma, glioma) and compared to non-tumor tissues adjusting for age and sex. Embryonal tumors had the most extensive differential 
hypohydroxymethylation with 12,593 loci (40\%) losing $5 \mathrm{hmC}$ (FDR $<0.1$, (Fig. 5a). We subset these loci to CpGs with an FDR $<0.02(n=1832)$ and performed a GREAT analysis. The previously observed response to steroid pathways was maintained, and pathways related to mTOR regulation emerged (Additional file 4). The extent of differential hydroxymethylation in ependymomas compared with non-tumor tissues was lower than that of embryonal tumors $(n=1630$ DHMR, FDR $<0.1$, Fig. $5 b)$. We did not observe significant differential hydroxymethylation among gliomas compared with non-tumor samples' DHMRs (Fig. 5c). Though gliomas did not appear to be significantly differentially hydroxymethylated as compared to controls, we questioned whether this was due to the inherent bias of subsetting our analysis to the high $5 \mathrm{hmC}$ loci. Therefore, we ran the linear model on all loci in the array and found 25, 147 differentially hydroxymethylated CpGs (Additional file 6: Fig. S6). We repeated GREAT analysis for ependymomas with loci with an $\mathrm{FDR}<0.1 \quad(n=1630)$ and gliomas with an $\mathrm{FDR}<0.2$
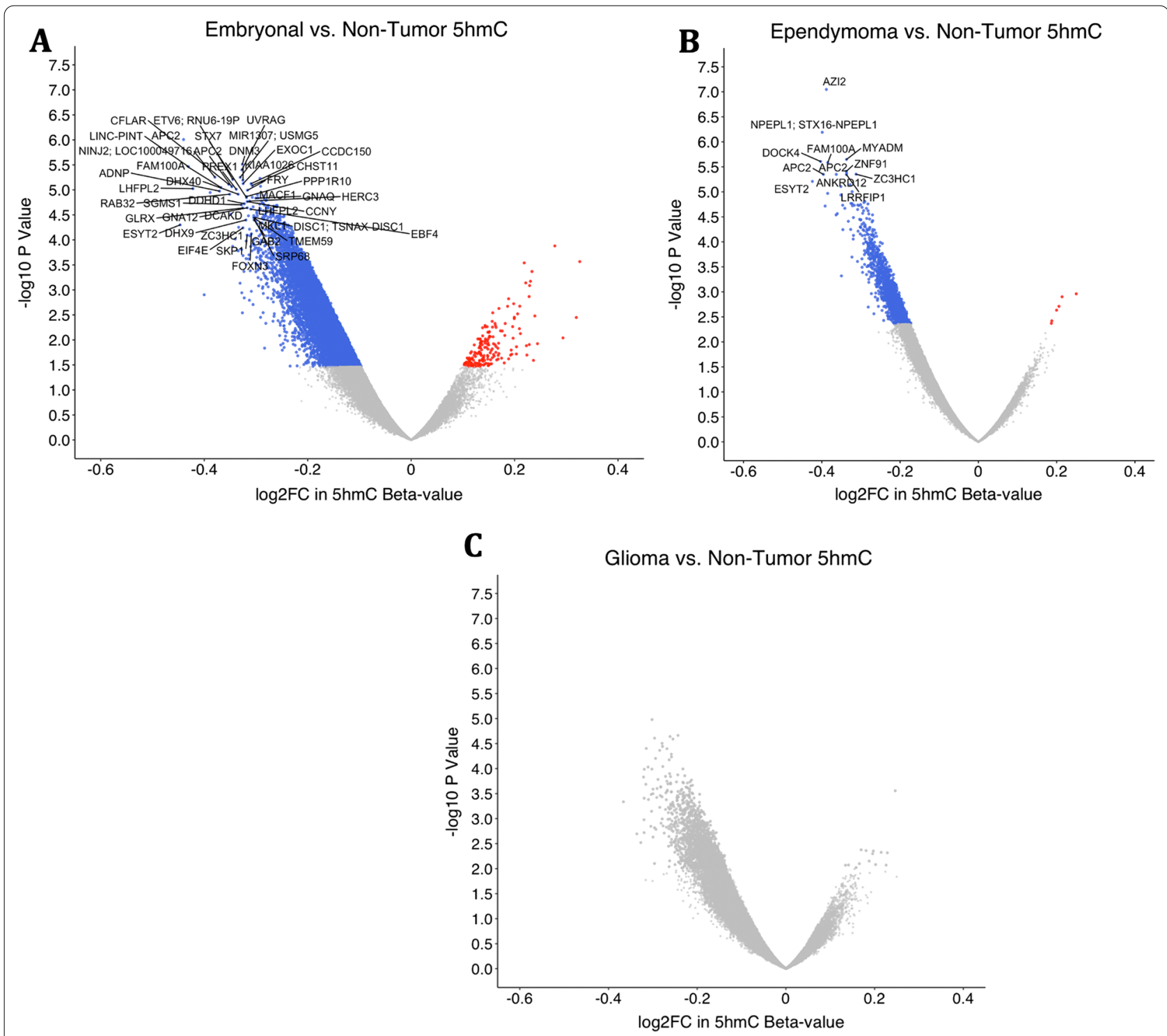

Fig. 5 Results from linear models comparing CpG hydroxymethylation in each tumor subtype to non-tumors. Blue represents loss of $5 \mathrm{hmC}$ and red gain of $5 \mathrm{hmC}$ by tumor as compared to controls. a Volcano plot of differentially hydroxymethylated regions in a comparison between embryonal and control samples. Colored points represent CpGs that are differentially hydroxymethylated with an adjusted $P$ value (FDR) less than 0.1 . 15,072 CpGs of the 37,173 high 5hmC CpGs were significantly differentially hydroxymethylated with an FDR less than 0.1. b Volcano plot comparing ependymomas to non-tumors. Colored points represent an FDR less than 0.1. c Comparison of gliomas to controls. No points reached statistical significance 
( $n=637$ ) (Additional file 4). Response to corticosteroid pathways was consistently present across all tumor types.

\section{$5 \mathrm{hmC}$ and survival}

Prior publications identified an association between decreased total $5 \mathrm{hmC}$ and survival in CNS tumors with mixed results $[13,40]$. Here, we used a modelbased clustering method, recursively partitioned mixture model (RPMM) [51], to discern profiles of $5 \mathrm{hmC}$ and investigate the association of profiles with survival. Applying RPMM to the top 10,000 most variable sites in tumor samples yielded two classes of tumors, which correlated with mean $5 \mathrm{hmC}$ levels $(P=2.735 \mathrm{E}-05$, Kruskal-Wallis rank-sum test, $d f=1)$, and we then defined the two classes as high $5 \mathrm{hmC}$ and low $5 \mathrm{hmC}$. A Chi-squared test evaluating the relationship between RPMM clusters and grade did not reach our threshold for statistical significance with a $P$ value of 0.099 . Similarly, we did not have sufficient evidence to conclude an association of RPMM cluster with tumor type (Embryonal OR 0.68, CI 0.05-6.03,
$P>0.99$, Ependymoma OR 1.68, CI 0.23-12.42, $P=0.67$, Glioma OR 0.84, CI 0.14-4.98, $P>0.99$ ) or subject age (Kruskal-Wallis rank-sum test $P=0.26$, $d f=1$ ). With the exception of the glioblastoma sample which was excluded from our survival analysis due to lack of follow up and one anaplastic ganglioglioma, all gliomas were low grade. All embryonal tumors in our study were grade 4 and only 2 of 8 ependymomas were high grade. Despite this, there was relatively equal distribution of tumor types in high and low $5 \mathrm{hmC}$ clusters. The low $5 \mathrm{hmC}$ cluster was composed of $25 \%$ embryonal tumors, $25 \%$ ependymoma, $50 \%$ gliomas while the high $5 \mathrm{hmC}$ class had $18 \%$ embryonal, 36\% ependymoma, $45 \%$ gliomas (Fig. 6). Then, we fit two independent multivariable Cox proportional hazards models for survival and recurrence adjusting for patient sex and tumor type, for the outcomes of death and recurrence. The low $5 \mathrm{hmC}$ cluster was associated with an increased hazard of death (HR 6.47, 95\% CI 0.79-53.2, $P=0.08$, concordance index $=0.84$, Cox proportional hazards regression) and recurrence

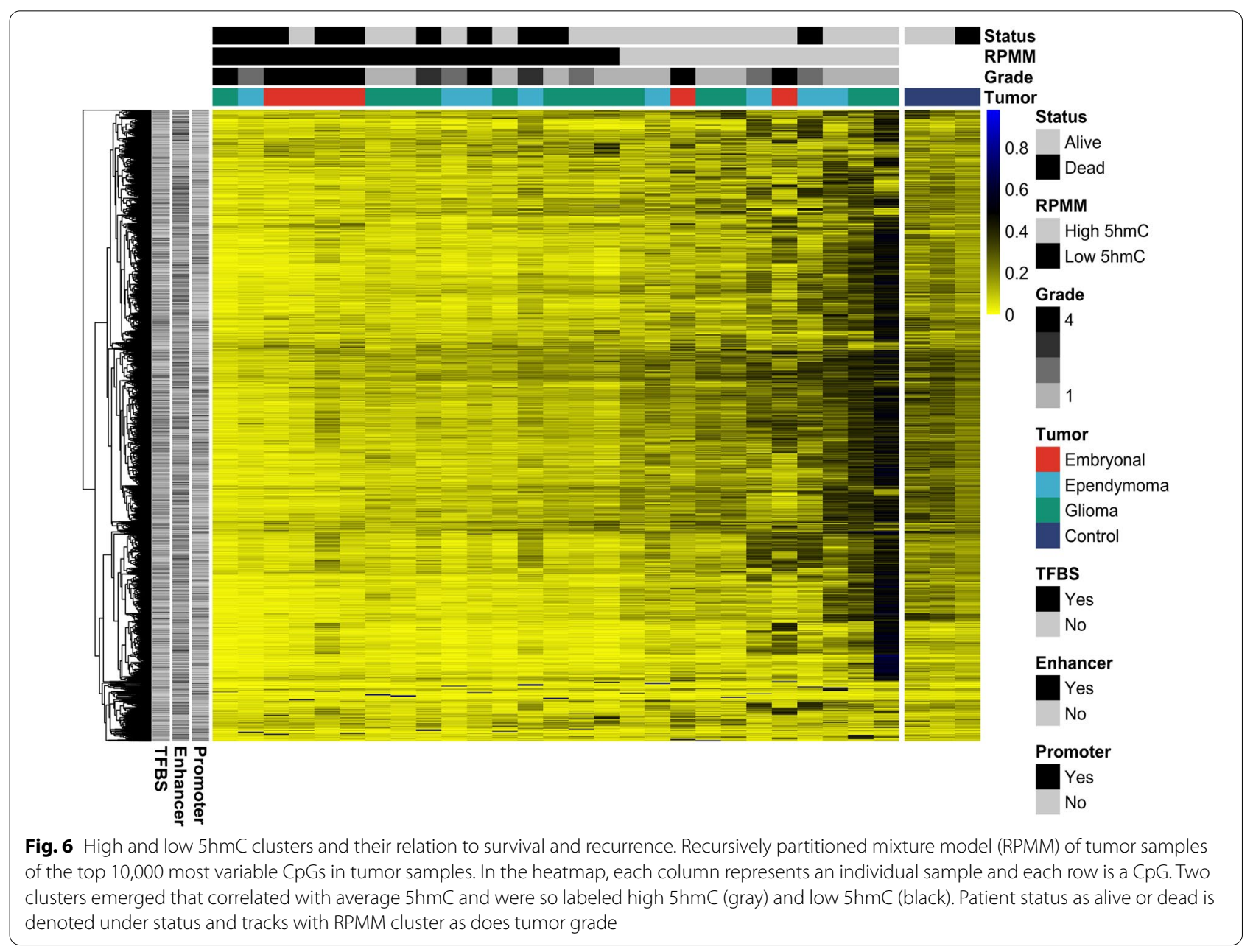


Table 2 Cox proportional hazard models of survival by RPMM 5hmC cluster membership

\begin{tabular}{|c|c|c|c|c|c|}
\hline \multicolumn{3}{|l|}{$A$} & \multicolumn{3}{|l|}{ B } \\
\hline \multicolumn{3}{|c|}{ Cox proportional hazard ratios for survival ${ }^{a}$} & \multicolumn{3}{|c|}{ Cox proportional hazard ratios for recurrence ${ }^{b}$} \\
\hline Variable & $\mathrm{HR}(95 \% \mathrm{Cl})$ & $P$ value & Variable & $\mathrm{HR}(95 \% \mathrm{Cl})$ & $P$ value \\
\hline Age & $0.97(0.83-1.1)$ & 0.70 & Age & $0.93(0.80-1.1)$ & 0.30 \\
\hline Sex & & & Sex & & \\
\hline Female & 1.0 (referent) & & Female & 1.0 (referent) & \\
\hline Male & $0.73(0.12-4.3)$ & 0.73 & Male & $0.70(0.20-2.4)$ & 0.58 \\
\hline Tumor type & & & Tumor type & & \\
\hline Glioma & 1.0 (referent) & & Glioma & 1.0 (referent) & \\
\hline Ependymoma & $3.93(0.54-28.6)$ & 0.18 & Ependymoma & $6.63(1.21-36.3)$ & 0.03 \\
\hline Embryonal & $4.95(0.63-38.9)$ & 0.13 & Embryonal & $4.27(0.76-24.1)$ & 0.10 \\
\hline RPMM cluster & & & RPMM cluster & & \\
\hline High 5hmC cluster & 1.0 (referent) & & High $5 \mathrm{hmC}$ cluster & 1.0 (referent) & \\
\hline Low $5 \mathrm{hmC}$ cluster & $6.47(0.79-53.2)$ & 0.08 & Low 5 hmC cluster & $4.83(0.95-24.6)$ & 0.06 \\
\hline
\end{tabular}

${ }^{a}$ Multivariate Cox proportional hazard ratios and confidence interval for survival based on RPMM cluster membership adjusted for age, sex, and tumor type demonstrated an increased hazard of death for the low 5hmC RPMM cluster (HR 6.47, 95\% Cl 0.79-53.2, $P=0.08$ )

${ }^{b}$ Multivariate cox proportional hazard ratio for recurrence based on RPMM cluster membership adjusting for age, sex, and tumor type demonstrated an increased hazard of recurrence for the low $5 \mathrm{hmC}$ cluster (HR $4.83,95 \% \mathrm{Cl} 0.95-24.6, P=0.06$ )

(HR 4.83, 95\% CI 0.95-24.6, $P=0.06$, concordance index $=0.79$ ) (Table 2). Applying the model but adjusting for grade demonstrated similar trends of improved prognosis for patients with high $5 \mathrm{hmC}$ tumors, but were underpowered (Additional file 6: Table S4 and Fig. S7).

In order to compare our results to more common methods of correlating $5 \mathrm{hmC}$ with survival, we constructed an index representing the total $5 \mathrm{hmC}$ content. The index is defined by averaging beta-values of all CpGs $(n=743,461)$ and assigning a sample to a "High Total $5 \mathrm{hmC}$ " group if the mean was greater than the 50th percentile (i.e., the sample mean $5 \mathrm{hmC}$ level was greater than 0.035). The results were consistent with the RPMM method described above, with the low $5 \mathrm{hmC}$ group having poorer survival (log-rank $P=0.08$, concordance index 0.75) (Additional file 6: Fig. S8). In a multivariable Cox proportional hazards model adjusted for sex and tumor type, the low total $5 \mathrm{hmC}$ group was associated with increased risk of recurrence though this did not reach statistical significance (HR 3.6, CI 95\% 0.9-14.5, $P=0.07)$ and death (HR 3.4, CI 95\% 0.69-16.5, $P=0.14$ ).

Applying principal component analysis (PCA) of this subset of CpGs, revealed discernible clusters that overlapped substantially with the different tumor types (Additional file 6: Fig. S9a). PCA also showed an overlap of clusters with survival (Additional file 6: Fig. S9b). Using methylation beta values at these loci also yielded discernible cluster separation by survival emphasizing the potential clinical relevance of our CpGs (Additional file 6: Fig. S9c).

\section{5hmC and methylation-based classification systems}

We next assessed the robustness of epigenetic CNS tumor classification methods to array data that is $5 \mathrm{mC}$ specific. Molecular neuropathology accepts bisulfitetreated IDAT files from CNS tumors and offers a list of diagnosis with best fit. We submitted IDAT files from both bisulfite and oxidative bisulfite-treated samples were submitted to this methylation-based CNS tumor classification system [36]. Despite high-quality data from fresh frozen specimens provided from the bisulfite-treated IDAT files, the molecularneuropathology.org tool could not classify $26 \%(7 / 27)$ of our tumors labeling them "Not Defined" or "Control tissue." Undefined tumors included those with histopathologic diagnoses both common such as medulloblastoma and subependymoma as well as rare such as dysplastic gangliocytoma, desmoplastic and anaplastic ganglioglioma. Of particular interest, when the OxBS-treated IDAT files, representing $5 \mathrm{mC}$-specific signal, from the same tumors were submitted, 26\% (7/27) of the samples switched diagnoses (Table 3), suggesting that hydroxymethylated CpGs are included in the Capper et al. random-forest-based classification system. In addition, we used a pediatric focused methylation-based classification tool called MethPed [34, 35] to investigate $5 \mathrm{mC}$-specific signal in tumor classification. While this tool only left the dysplastic ganglioglioma as undefined, it over diagnosed our samples as glioblastomas. Many 
Table 3 Methylation-based classification systems and tumor type predictions

\begin{tabular}{|c|c|c|c|c|c|}
\hline Capper methylation-based classification tool & $\mathrm{BS}(n)$ & OxBS (n) & MethPed methylation-based classification tool & $\mathrm{BS}(n)$ & OxBS $(n)$ \\
\hline Glioma & 11 & 9 & Glioma & 15 & 18 \\
\hline Anaplastic pleomorphic xanthoastrocytoma & 1 & 1 & Pilocytic astrocytoma & 7 & 11 \\
\hline CNS high-grade neuroepithelial tumor with MN1 alteration & 1 & 1 & Glioblastoma & 8 & 7 \\
\hline Pilocytic astrocytoma & 6 & 6 & & & \\
\hline Glioma, IDH mutant & 1 & 1 & & & \\
\hline $\begin{array}{l}\text { Low-grade glioma, dysembryoplastic neuroepithelial } \\
\text { tumor }\end{array}$ & 2 & & & & \\
\hline Ependymoma & 5 & 4 & Ependymoma & 5 & 5 \\
\hline Ependymoma, myxopapillary & 1 & 1 & & & \\
\hline Ependymoma, posterior fossa group A & 3 & 2 & & & \\
\hline Ependymoma, RELA fusion & 1 & 1 & & & \\
\hline Embryonal & 4 & 4 & Embryonal & 6 & 4 \\
\hline \multirow[t]{3}{*}{ Medulloblastoma group 3 and 4} & 4 & 4 & Medulloblastoma SHH & 1 & 1 \\
\hline & & & Medulloblastoma group 3 and 4 & 4 & 3 \\
\hline & & & Embryonal tumor with multilayered rosettes & 1 & \\
\hline Undefined & 7 & 10 & Undefined & 1 & 0 \\
\hline Not defined & 6 & 6 & & & \\
\hline Control tissue & 1 & & & & \\
\hline Plexus tumor & & 3 & & & \\
\hline CNS neuroblastoma with FOXR2 activation & & 1 & & & \\
\hline
\end{tabular}

a Sample IDAT files from both BS and OxBS-treated tissues were submitted to methylation-based CNS tumor classification systems: molecular neuropathology and MethPed. The table above demonstrates how classifications switched in pediatric tumors based on the classification system and the files submitted

of the samples that the Capper classification tool left undefined, MethPed predicted to be glioblastomas. The MethPed classification of OxBS derived $5 \mathrm{mC}$-specific data resulted in 24/27 (89\%) of tumors switching diagnoses. Further, when we tested the Capper Classification system on $5 \mathrm{mC}$-specific adult glioblastoma data from our prior work on hydroxymethylation [13] 73\% $(22 / 30)$ of tumors changed diagnoses, suggesting that $5 \mathrm{hmC}$ plays an even greater role in adult glioblastoma classification than pediatric tumors. Most tumors were reclassified from IDH wildtype glioblastoma to "control tissue, inflammatory tumor microenvironment." To assess whether tumor purity affected the diagnosis of the classification systems, we derived purity estimates with the Infinium Purify [52] method. There was no association between class switching and tumor purity for either the MethPed (ANOVA, $P=0.7$ ) or Capper classification systems (ANOVA, $P=0.361$ ).

To compare overlap of our high $5 \mathrm{hmC}$ with CpGs used in the Capper unsupervised analysis, we explored the overlap between the most variable CpGs in the Capper dataset and the high $5 \mathrm{hmC}$ CpGs in our pediatric cohort and adult glioblastomas. We isolated 31,476 with a S.D $>0.228$ to capture an approximation of the 32,000 most variably methylated probes mentioned in the original paper's methods. We then reprocessed the pediatric and adult samples to identify the top 5\% most hydroxymethylated CpGs in both groups. 723 of the 19,353 (3.7\%) pediatric high $5 \mathrm{hmC}$ CpGs overlapped with the Capper set. Consistent with the large proportion of tumors that switched classes for adult glioblastomas, 4979 of the 19,353 (26\%) high 5hmC CpGs from that data set overlapped with the Capper classification CpG set (Fig. 7). With regard to the MethPed probes, the $900 \mathrm{CpGs}$ used by their classification system were published and we found that 45 of the 900 probes overlapped with the high $5 \mathrm{hmC} \mathrm{CpGs} \mathrm{in} \mathrm{the} \mathrm{pediatric} \mathrm{study.}$

\section{Discussion}

Childhood nervous system tumors have a relatively low incidence of mutations. Common variations tend to be related to epigenetic mechanisms such as chromatin remodeling [53-55]. DNA methylation has been explored as a potential driver, and our results delineate the unique role of $5 \mathrm{hmC}$ from $5 \mathrm{mC}$ in CNS tumor pathology. We demonstrate that $5 \mathrm{hmC}$ is lost in tumors in a locus-specific pattern and that these sites are hypermethylated. This mirrors findings in adult glioblastomas, which undergo a similar exchange of $5 \mathrm{hmC}$ for $5 \mathrm{mC}$ [23]. CpG islands that were specifically affected by this pattern include $A P C 2$, a regulator of WNT signaling pathway, and $K D M 2 A$, a histone demethylase. 


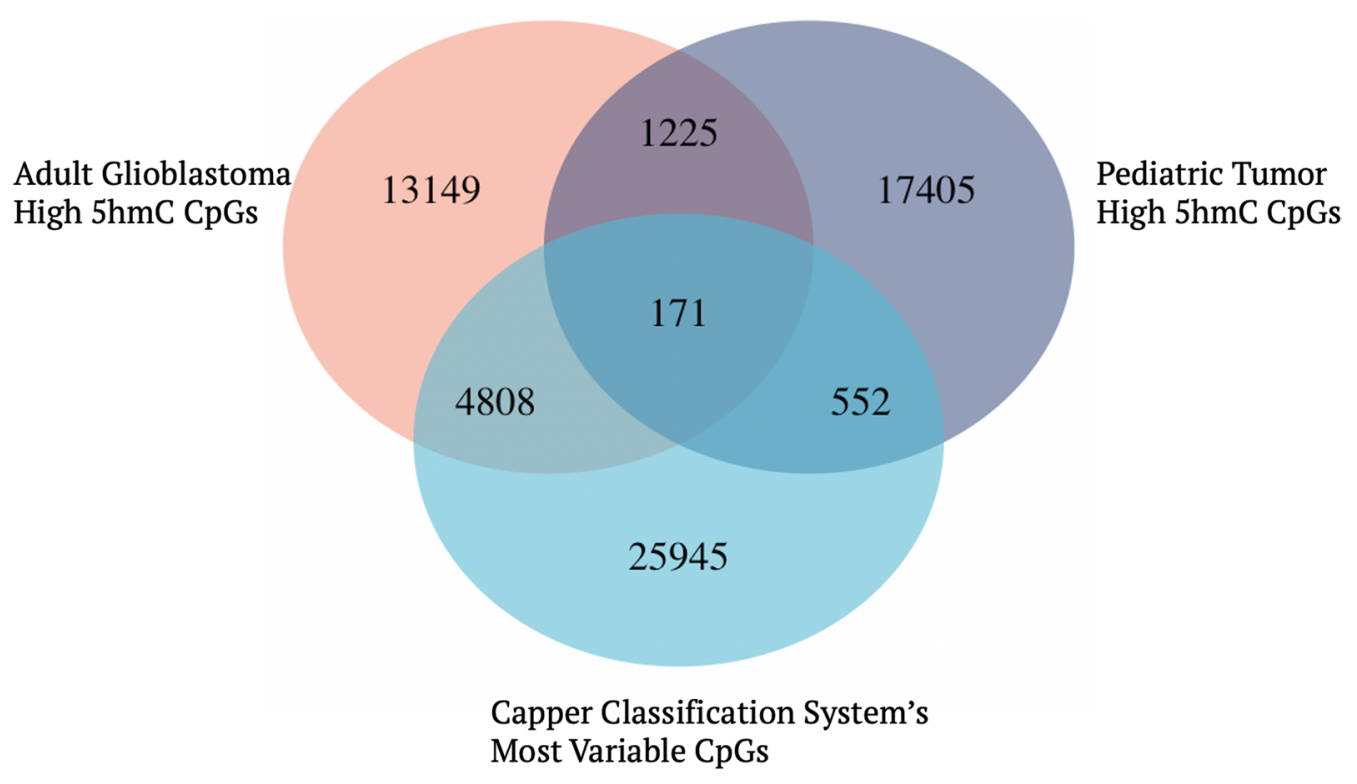

Fig. 7 Venn diagram of shared CpGs in adult and pediatric tumor high $5 \mathrm{hmC}$ loci and methylation-based classification system's most variable loci. The overlap of high $5 \mathrm{hmC}$ sites identified in pediatric tumors and adult glioblastomas with the most variable CpGs in the Capper data set with high $5 \mathrm{hmC}$ loci in pediatric and adult CNS tumors. There was considerably greater overlap between adult glioblastoma's high $5 \mathrm{hmC}$ loci and the Capper data sets most variable CpGs (18\%) than with the pediatric tumors' high 5hmC CpGs (2\%)

CNS tumor classification systems such as MethPed and Molecular Neuropathology rely on bisulfite-treated data that do not distinguish between $5 \mathrm{hmC}$ and $5 \mathrm{mC}$. Our study suggests for the first time that $5 \mathrm{hmC}$ affects methylation-based CNS tumor classification systems as OxBS files which contain $5 \mathrm{mC}$ levels changed diagnoses. Oxidative bisulfite treatment enabled us to resolve this relationship, whereas bisulfite only treated samples-which measure the sum of $5 \mathrm{hmC}$ and $5 \mathrm{mC}$-demonstrated hypomethylation in tumors as compared to controls. This was independent of tumor purity and more pronounced in adult glioblastomas than pediatric tumors. We postulate that given the high rate of change in classification with the OxBS data that $5 \mathrm{hmC}$ does play a role in the classification of these tumors but that the CpGs used to assign pediatric CNS tumor diagnosis are not necessarily those with the highest $5 \mathrm{hmC}$ levels. This is in contrast with adult glioblastomas which not only demonstrated a higher rate of change but also had $26 \%$ of the high $5 \mathrm{hmC}$ loci in the most variable loci of the Capper dataset.

It appears that loci that are critical to tumor classification are not necessarily the most hydroxymethylated due to little overlap of loci that are used in these classification systems with our list of high $5 \mathrm{hmC} \mathrm{CpGs.} \mathrm{Our} \mathrm{study}$ as well as previously conducted projects have focused on the most hydroxymethylated loci as these tend to have the clearest signals and are less likely to be attributable to noise alone. However, our findings motivate further examining loci with median levels of $5 \mathrm{hmC}$. Our results reinforce the importance of a more nuanced approach to the study of cytosine modifications in CNS tumors, and offer new opportunities to refine classification approaches that benefit clinicians and patients [36].

Though 5-hydroxymethylation in pediatric brain tumors has been investigated, studies to date have not been on a genome-scale at a locus resolution. An association between $5 \mathrm{hmC}$ and anaplasia was established using immunohistochemistry (IHC) to examine $5 \mathrm{hmC}$ in all WHO classifications of brain tumors [56]. Our study adds to existing literature that $5 \mathrm{hmC}$ accumulates in super-enhancers and suggests a link between a loss of $5 \mathrm{hmC}$ and anaplasia [56]. Recently, Wu et al. showed high $5 \mathrm{hmC}$ levels were associated with worse survival in pediatric posterior fossa ependymomas [40]. However, as this study was conducted using IHC, it remains unclear where in the genome this hyperhydroxymethylation was taking place. In line with previous studies, we observed that the loss of $5 \mathrm{hmC}$ in tumors was associated with shorter overall survival and time to recurrence [13, 22]. A nucleotide level analysis allowed us to identify enrichment of high $5 \mathrm{hmC}$ loci in genes critical to normal craniofacial and neurodevelopment further strengthening the link between these tumors and developmental neurobiology [57]. We also found that differentially hypohydroxymethylated CpGs are enriched in molecular pathways that are frequently connected to childhood brain tumors, 
particularly those related to WNT signaling and betacatenin binding, implicating such genes as $A P C 2, W N T 4$, WNT11, WNT5B, SHH, and BCL9. 5hmC loss in WNT has been associated with other tumors such as melanoma and colorectal cancer $[26,30]$.

The tendency of $5 \mathrm{hmC}$ to accumulate at $5^{\prime}$ splicing sites in the exon-intron boundary has been suggested as a link between the epigenetic marker and alternative splicing [58]. We found that not only did our high $5 \mathrm{hmC}$ sites localize to $5^{\prime}$ untranslated regions in concordance with previous work but that these loci were enriched in genes implicated in posttranslational regulation of gene expression such as the DICER1, AGO2, and EIF2C2. The association between $5 \mathrm{hmC}$ and CTCF, a methylationsensitive transcription factor linked to alternative splicing and RNA polymerase II regulation, has also been reported in embryonal cells $[12,59]$. Increased $5 \mathrm{hmC}$ levels have been tied to reduced binding of nucleosomes to DNA and reduced CTCF attachment [60]. $5 \mathrm{hmC}$ has been shown to oscillate at 150 nucleotides, the length of nucleosome wound DNA, and $5 \mathrm{hmC}$ has been suggested as a linker binding CTCF to DNA [61].

Due to the extreme rarity of pediatric brain tumors, our study is limited by the sample size. Additionally, our study consists primarily of medulloblastomas, pilocytic astrocytomas and ependymomas based on the prevalence of these tumors therefore generalizable largely to these subtypes. Larger studies would need to be done in order to capture the true effect of $5 \mathrm{hmC}$ on pediatric CNS tumors. In the future, a larger multi-institutional study is warranted to narrow the confidence interval for $5 \mathrm{hmC}$ association with patient outcomes and increase power for differential hydroxymethylation analysis in tumor subgroups. We did not observe significant differential hydroxymethylation among gliomas compared with non-tumor samples, which is not unlikely a reflection of the heterogeneity of this tumor class. These tumors were diagnosed prior to the WHO 2016 Classification of CNS tumors [3] with the updated molecular studies that it entailed, and it is possible that some of these tumors would be diagnosed differently if the most recent criteria were applied. However, histopathological re-review was conducted for all samples. Though potential variation in the proportion of non-tumor tissue content may have contributed to more proximal clustering with non-tumor samples and higher total $5 \mathrm{hmC}$ levels, the observed association of $5 \mathrm{hmC}$ with survival and recurrence was robust to this potential variation. Including multiple types of pediatric central nervous system tumors, both rare and common, and comparing tumor tissue to control samples enhances the generalizability of our findings. In the future, $5 \mathrm{hmC}$ measures have promise for clinical applications guiding both diagnosis and treatment.
There is evidence that treatment affects the samples $5 \mathrm{hmC}$ levels. All but two of our samples were from patients diagnosed when chemotherapy regimens were largely give in an adjuvant setting and are expected to be chemotherapy naïve. One glioblastoma sample taken at the time of autopsy timing of treatment was unknown. Aside from chemotherapy, children with CNS tumors are almost always given dexamethasone for tumor edema and this was reflected in our results. For instance, GREAT analysis of differentially hydroxymethylated regions in the tumor versus non-tumor comparison demonstrated the clustering of DHMRs in steroid response pathways. This is likely due to the treatment of most CNS tumors with dexamethasone in order to reduce edema and the risk of herniation. This relationship was present across all tumor subtypes. $5 \mathrm{hmC}$ has been associated with steroidinduced osteonecrosis and endometriosis, and our data provide further evidence of the connection between the medication and the epigenomic marker [62, 63].

\section{Conclusions}

To our knowledge, we describe for the first time a genome-wide cytosine-specific analysis of $5 \mathrm{hmC}$ in three classes of childhood CNS tumors: embryonal, glioma, and ependymoma. Super-enhancer targeting by $5 \mathrm{hmC}$ in all three classes of tumors was identified, and genes commonly implicated in pediatric CNS tumors were differentially hypohydroxymethylated. We demonstrated that distinguishing methylation and hydroxymethylation is critical in identifying tumor-related epigenetic changes.

\section{Materials and methods}

\section{Study population and samples}

Pathologically confirmed fresh-frozen primary CNS tumor specimens from 27 unique individuals were identified; they included thirteen gliomas, eight ependymomas, and six embryonal tumors. All patients were treated at Dartmouth Hitchcock Medical Center. Samples were collected from patients who had provided consent for the use of tissues for research purposes as approved by the committee for the protection of human subjects (Institutional Review Board). Detailed information about each patient, including demographics, tumor histopathology, survival, time to recurrence, metastasis, chemotherapy and radiation regimens, was collected from the electronic medical record system. Pathologic re-review confirmed histopathologic tumor type and grade for all cases according to the 2016 WHO classification of CNS Tumors [3]. A table with complete patient information, including survival and treatment received is available (Additional file 1). Five patients had greater than one sample with two patients each having four samples available. Four fresh-frozen non-tumor brain specimens 
from children ranging in age from newborn to 11 years were selected as control tissues. Three tissues were from patients with epilepsy who underwent surgical resection and were used in our analyses. One tissue was excluded from analysis as it was from a non-immune hydrops fetalis subject at only $216 / 7$ weeks gestational age.

\section{DNA extraction and modification}

Tumor DNA was extracted from fresh frozen tumor tissue using DNeasy Blood and Tissue Kit (Qiagen) following the manufacturer's instructions. Approximately, 1-15 mg of tumor tissue was used for DNA extraction. DNA was subjected to tandem bisulfite and oxidative bisulfite (OxBS) conversion using the TruMethyl OxBs module kit (Cambridge Epigenetix-Nugen), according to the TrueMethyl protocol M01481, Revision v2.

\section{TERT promoter and $H 3 F 3 A$ sequencing}

Tumor samples were sequenced for TERT Promoter (C2505, C280T) and H3F3A mutations status (K27M, G34) using PCR amplification and Sanger sequencing. $\sim 10 \mathrm{ng}$ of genomic DNA per sample was amplified (see Appendix for the list of primers). For H3F3A, OneTaq Hot Start $2 \times$ Master Mix with standard buffer was used (NEB). PCR cycle parameters were as follows: denaturation at $95{ }^{\circ} \mathrm{C}$ for $30 \mathrm{~s}$, followed by 35 cycles of $94{ }^{\circ} \mathrm{C}$ for $30 \mathrm{~s}, 48{ }^{\circ} \mathrm{C}$ for $30 \mathrm{~s}, 68{ }^{\circ} \mathrm{C}$ for $1 \mathrm{~min}$, with the final extension at $68{ }^{\circ} \mathrm{C}$ for $5 \mathrm{~min}$. Amplicons were visualized in Agarose Gel each time. For TERT Promoter, OneTaq Hot Start 2X Master Mix with GC buffer (NEB) was used. Cycle parameters were as follows: denaturation at $94{ }^{\circ} \mathrm{C}$ $30 \mathrm{~s}$, followed by 35 cycles of $94{ }^{\circ} \mathrm{C} 30 \mathrm{~s}, 59^{\circ} \mathrm{C}$ for $30 \mathrm{~s}$, $68{ }^{\circ} \mathrm{C}$ for $1 \mathrm{~min}$, with a final extension at $68^{\circ} \mathrm{C}$ for $5 \mathrm{~min}$. DNA was purified using the Qiagen PCR purification kit prior to visualization on an agarose gel. Primer sequences used can be found in Additional file 5 .

\section{Cytosine modification measures}

Illumina Human Methylation EPIC Beadchips were loaded with sample DNA in a randomized fashion with equal distribution of all tumor subtypes and non-tumor samples in each chip in order to eliminate batch effects. Beadchips were read using the Illumina iScan reader, and sample intensity data (IDAT files) was generated. Normalization and background correction from each of the BS and OxBS converted samples was performed using the Funnorm procedure available in the R/Bioconductor package minfi (version 1.30.0) [64]. Before analysis, we removed $\mathrm{CpG}$ sites on sex chromosomes using the updated Illumina EPIC annotation file (R/Bioconductor package IlluminaHumanMethylationEPICanno. ilm10b4.hg19) and SNP probes identified in the EPIC array [65]. To estimate methylated, hydroxymethylated, and unmethylated proportions of cytosine, we applied the OxyBS algorithm which takes bisulfite-treated data which includes both $5 \mathrm{mC}$ and $5 \mathrm{hmC}$ levels and approximates $5 \mathrm{hmC}$ signal intensity by subtracting oxidative bisulfite-treated data which provides only $5 \mathrm{mC}$ signal intensities [39]. Through this method, a total of 743,461 probes were left for analysis. Array data analysis was conducted in $\mathrm{R}$ version 3.6.1 [66].

\section{Statistical analyses}

We identified and subset our data to the 5\% $(37,173 / 743,461)$ most highly hydroxymethylated CpG sites (high $5 \mathrm{hmC}$ loci), as determined by the median, across all tumor types. We performed Fisher's exact tests for enrichment of these 37,173 consistently hydroxymethylated $\mathrm{CpGs}$ in $\mathrm{CpG}$ islands, shores, and shelves against the 743,461 CpG universe. For gene promoter, exon, and intron regions and regulatory elements, we used a Cochran-Mantel-Haenszel test to assess enrichment stratifying by probe type. The Phantom 5 enhancer annotation (available in the Illumina EPIC annotation file) was used to map CpGs in enhancer regions. Coordinates for super-enhancers were downloaded from the dbSuper database [43]. We examined the 486 genes in which these high $5 \mathrm{hmC} \mathrm{CpGs}$ were enriched using the UCSC reference gene names in the Illumina annotation file. We selected for genes that had at least 10 CpGs represented in the EPIC array. We used the genomic coordinates of these high $5 \mathrm{hmC}$ CpGs in enriched genes as a query set of regions and submitted to Genomic Regions Enrichment of Annotations Tool (GREAT) analysis. We tested for enrichment against the background of the 743,173 CpGs in the EPIC array used in our analyses.

\section{Analysis of CpG-specific associations}

Differential hydroxymethylation status between tumor and non-tumor brain tissue at the CpG loci in our data set was determined through multivariable linear models for microarray data (limma) [67]. Models were adjusted for subject age and sex and applied to $5 \mathrm{hmC} \beta$-values. Benjamini-Hochberg correction was used to adjust for multiple testing. We examined whether there were differences between tumors and non-tumors in $5 \mathrm{hmC} \mathrm{lev-}$ els within the top 5\% most hydroxymethylated CpGs $(37,173)$. We applied the same model to each tumor subtype and compared them to non-tumor samples in order to determine if the results changed when stratified.

To explore if the loss of tumor hydroxymethylation in high $5 \mathrm{hmC}$ loci was limited to specific gene sets, we selected CpGs that were differentially hypohydroxymethylated with negative $\log$ fold change $(\log -\mathrm{FC}<0)$ and FDR less than 0.1 (726 CpGs) and queried the GREAT software [68]. To test if these loci were associated with 
transcription factor binding sites or sites of histone modifications, we interrogated the ENCODE and Roadmaps database using the LOLA R/Bioconductor package [69]. We tested for enrichment in CpG islands, shores and shelves using a Fisher's exact test against the 37,173 high $5 \mathrm{hmC}$ loci. We used the same universe to test for enrichment of DHMRs in regulatory regions and super-enhancers using a Cochran-Mantel-Haenszel test.

\section{Survival analysis}

Recursively partitioned mixture model (RPMM) has been used for clustering DNA methylation and hydroxymethylation data to identify classes of tumors based on beta values [51]. Here, we applied RPMM to the top 10,000 most variable $\mathrm{CpGs}$ across tumors as determined by variance, and the resulting clustering solution contained two distinct clusters, defining a low and a high $5 \mathrm{hmC}$ cluster. A multivariable Cox proportional hazards model adjusting for age at diagnosis, sex, and tumor type was used to determine if cluster designation correlated with survival. Although one patient in our cohort had a diagnosis of glioblastoma, the sample was collected at autopsy and no clinical information was available. Therefore, due to the lack of follow-up, we excluded this patient from our survival analysis.

\section{Evaluation of $5 \mathrm{hmC}$ in methylation-based classification systems}

Although the Capper classification system does not disclose the list of CpGs it uses to predict tumor type, we downloaded and processed the files from their experiment and isolated 31,476 with a S.D $>0.228$ to capture an approximation of the 32,000 most variably methylated probes they state were used in their methods. In order to evaluate overlap of adult and pediatric tumors' high $5 \mathrm{hmC}$ loci with the capper data set, we used IDAT files previously published experiments examining $5 \mathrm{hmC}$ levels in adult glioblastomas [13], reprocessed the files with ours and re-subset the complete dataset (encompassing both pediatric and adult data) to the CpGs with the top 5\% hydroxymethylation levels. This included 19, 353 CpGs. Infinium Purify [52] was used to determine tumor purity from methylation levels and publically available tumor IDAT files [36] were downloaded to provide more robust predictions.

\section{Abbreviations}

5caC: 5-Carboxylcytosine, an oxidized form of 5-methylcytosine; 5fC: 5-Formylcytosine, a derivative of 5-methylcytosine; $5 \mathrm{hmC}$ : 5-Hydroxymethylation, an oxidized form of DNA methylation; 5mC: 5-Methylcytosine, a methyl $(\mathrm{CH} 3)$ group added to the fifth carbon in cytosine found commonly in DNA. Alterations in methylation are commonly found in cancer pathologies; Cl: Confidence interval; CNS: Central nervous system which includes the brain and spinal cord; HR: Hazard ratio; IDH: Isocitrate dehydrogenase. A gene commonly mutated in adult glioblastomas; OR: Odds ratio; PCA: Principal component analysis. A statistical method that finds a line of best fit given a collection of points; TSS: Transcription start site.

\section{Supplementary Information}

The online version contains supplementary material available at https://doi. org/10.1186/s13148-021-01156-9.

Additional file 1. Includes a list of all samples, patient demographics, location and histopathologic diagnosis of the tumor, duration of clinical follow-up, treatments received as well as any known or tested mutations.

Additional file 2. Gene List: List of genes with a high proportion of represented probes being hyperhydroxymethylated. Gene name, number of probes for each gene represented in the EPIC array, number of probes that qualified as high $5 \mathrm{hmC}$ and the calculated percentage. Results of GREAT analysis: Results of submitting the coordinates of the high $5 \mathrm{hmC}$ loci to Genomic Regions Enrichment of Annotations Tool (GREAT) analysis. Terms associated with biologic process, cellular components and molecular function are included as well as $P$ value and FDR Q-Values.

Additional file 3. Great Analysis of DHMRs: Results of submitting DHMR loci to GREAT analysis. DHMR Genes: Results of linear model testing for sites of differential hydroxymethylation between tumor and non-tumor samples. Data includes the associated genes, relation to island, chromosomes, logFC and adjusted $P$ value.

Additional file 4. Embryonal V NonTumor Ontologies: Results of GREAT analysis of DHMRs from a linear model comparing embryonal and nontumor samples. Ependymoma V NonTumor Ontologies: Results of GREAT analysis of DHMRs from a linear model comparing ependymomas and non-tumor samples. Glioma V NonTumor Ontologies: Results of GREAT analysis of DHMRs from a linear model comparing gliomas and non-tumor samples.

Additional file 5. List of primers and sequences used for sanger sequencing.

Additional file 6. Supplemental Figures and Tables.

\section{Authors' contributions}

All authors contributed to the study conception and design. NA and LNN procured the samples and clinical data. NA and LP carried out laboratory experiments. NA, CLP, YC performed statistical analyses. All authors analyzed and interpreted the results. NA wrote the manuscript with contributions and critical revisions from all authors. All authors read and approved the final manuscript.

\section{Funding}

This research was supported by a Prouty Pilot Award from the Norris Cotton Cancer Center at Dartmouth-Hitchcock Medical Center. National Institutes of Health R01CA216265, and P20GM104416/6369. Mr. Petersen was supported by the Burroughs-Welcome Fund: Big Data in the Life Sciences at Dartmouth. Ms. Azizgolshani was supported by the S.M. Tenney Fellowship at Dartmouth.

\section{Availability of data and materials}

The pediatric tumor and non-tumor microarray data (IDAT files) were deposited at the Gene Expression Omnibus (GEO) under GSE152561 (http://www. ncbi.nlm.nih.gov/geo/).

\section{Code availability}

R code used for all analyses and figures in the paper can be found at https:// github.com/Christensen-Lab-Dartmouth/PCNS_5hmC_06112020.

\section{Declarations}

\section{Ethics approval and consent to participate}

All participants provided consent prior to utilization of their tumor samples in this research. The use of tissues for research purposes was approved by the committee for the protection of human subjects (Institutional Review 
Board) at Dartmouth Hitchcock Medical Center and Dartmouth College. All studies were carried out in accordance with the principles of the Declaration of Helsinki.

\section{Consent for publication \\ Not applicable.}

\section{Competing interests}

The authors declare that they have no competing interests.

\section{Author details}

'Department of Molecular and Systems Biology, Geisel School of Medicine at Dartmouth, Lebanon, NH 03756, USA. ${ }^{2}$ The Dartmouth Institute for Health Policy and Clinical Practice, Dartmouth College, Lebanon, NH 03756, USA. ${ }^{3}$ Department of Epidemiology, Geisel School of Medicine at Dartmouth, Lebanon, NH 03756, USA. ${ }^{4}$ Department of Pathology, Dartmouth-Hitchcock Medical Center, Lebanon, NH 03756, USA. ${ }^{5}$ Department of Laboratory Medicine and Pathobiology, University of Toronto, Toronto, ON, Canada.

\section{Received: 6 June 2021 Accepted: 18 August 2021}

\section{Published online: 19 September 2021}

\section{References}

1. Johnson KJ, Cullen J, Barnholtz-Sloan JS, Ostrom QT, Langer CE, Turner MC, et al. Childhood brain tumor epidemiology: a brain tumor epidemiology consortium review. Cancer Epidemiol Biomark Prev. 2014;23:2716-36.

2. Moore BD. Neurocognitive outcomes in survivors of childhood cancer. J Pediatr Psychol. 2005;30:51-63.

3. Louis DN, Perry A, Reifenberger G, von Deimling A, Figarella-Branger D, Cavenee WK, The, et al. World Health Organization Classification of Tumors of the Central Nervous System: a summary. Acta Neuropathol. 2016;2016:803-20.

4. Glod J, Rahmea GJ, Kaur H, Raabee E, Hwang El, Israel MA. Pediatric brain tumors: current knowledge and therapeutic opportunities. J Pediatr Hematol Oncol. 2016;38:249-60.

5. Cavalli FMG, Remke M, Rampasek L, Peacock J, Shih DJH, Luu B, et al. Intertumoral heterogeneity within medulloblastoma subgroups. Cancer Cell. 2017;31:737-754.e6.

6. Mack SC, Taylor MD. Put away your microscopes: the ependymoma molecular era has begun. Curr Opin Oncol. 2017;29:443-7.

7. Bar EE, Lin A, Tihan T, Burger PC, Eberhart CG. Frequent gains at chromosome 7q34 involving BRAF in pilocytic astrocytoma. J Neuropathol Exp Neurol. 2008;67:878-87.

8. Ito S, Shen L, Dai Q, Wu SC, Collins LB, Swenberg JA, et al. Tet proteins can convert 5-methylcytosine to 5-formylcytosine and 5-carboxylcytosine. Science (80-). 2011;333:1300-3.

9. Yu M, Hon GC, Szulwach KE, Song CX, Zhang L, Kim A, et al. Base-resolution analysis of 5-hydroxymethylcytosine in the mammalian genome. Cell. 2012;149:1368-80.

10. Bhattacharyya S, Pradhan K, Campbell N, Mazdo J, Vasantkumar A, Maqbool $S$, et al. Altered hydroxymethylation is seen at regulatory regions in pancreatic cancer and regulates oncogenic pathways. Genome Res. 2017;27:1830-42.

11. Tsagaratou A, Äijö T, Lio CWJ, Yue X, Huang Y, Jacobsen SE, et al. Dissecting the dynamic changes of 5-hydroxymethylcytosine in T-cell development and differentiation. Proc Natl Acad Sci U S A. 2014;111:E3306-15.

12. Ficz G, Branco MR, Seisenberger S, Santos F, Krueger F, Hore TA, et al. Dynamic regulation of 5-hydroxymethylcytosine in mouse ES cells and during differentiation. Nature. 2011;473:398-404.

13. Johnson KC, Houseman EA, King JE, von Herrmann KM, Fadul CE, Christensen BC. 5-Hydroxymethylcytosine localizes to enhancer elements and is associated with survival in glioblastoma patients. Nat Commun. 2016;7:13177.

14. Wilkins OM, Johnson KC, Houseman EA, King JE, Marsit CJ, Christensen BC. Genome-wide characterization of cytosine-specific 5-hydroxymethylation in normal breast tissue. Epigenetics. 2019. https://doi.org/10.1080/ 15592294.2019 .1695332$.

15. Green BB, Houseman EA, Johnson KC, Guerin DJ, Armstrong DA, Christensen $B C$, et al. Hydroxymethylation is uniquely distributed within term placenta, and is associated with gene expression. FASEB J. 2016;30:287484. https://doi.org/10.1096/fj.201600310R.

16. Branco MR, Ficz G, Reik W. Uncovering the role of 5-hydroxymethylcytosine in the epigenome. Nat Rev Genet. 2012;13:7-13.

17. Kriaucionis S, Heintz N. The nuclear DNA base 5-hydroxymethylcytosine is present in purkinje neurons and the brain. Science (80-). 2009:324:929-30.

18. Khare T, Pai S, Koncevicius K, Pal M, Kriukiene E, Liutkeviciute Z, et al. 5 -hmC in the brain is abundant in synaptic genes and shows differences at the exon-intron boundary. Nat Struct Mol Biol. 2012;19:1037-43.

19. Spiers H, Hannon E, Schalkwyk LC, Bray NJ, Mill J. 5-hydroxymethylcytosine is highly dynamic across human fetal brain development. BMC Genomics. 2017;18:738. https://doi.org/10.1186/s12864-017-4091-x.

20. Dong E, Dzitoyeva SG, Matrisciano F, Tueting P, Grayson DR, Guidotti A. Brain-derived neurotrophic factor epigenetic modifications associated with schizophrenia-like phenotype induced by prenatal stress in mice. Biol Psychiatry. 2015;77:589-96.

21. Cheng Y, Li Z, Manupipatpong S, Lin L, Li X, Xu T, et al. 5-Hydroxymethylcytosine alterations in the human postmortem brains of autism spectrum disorder. Hum Mol Genet. 2018;27:2955-64.

22. Glowacka WK, Jain H, Okura M, Maimaitiming A, Mamatjan Y, Nejad R, et al. 5-Hydroxymethylcytosine preferentially targets genes upregulated in isocitrate dehydrogenase 1 mutant high-grade glioma. Acta Neuropathol. 2018;135:617-34. https://doi.org/10.1007/s00401-018-1821-3.

23. Raiber EA, Beraldi D, Martínez Cuesta S, Mclnroy GR, Kingsbury Z, Becq J, et al. Base resolution maps reveal the importance of 5-hydroxymethylcytosine in a human glioblastoma. npj Genomic Med. 2017;2:1-7.

24. Salgado C, Oosting J, Janssen B, Kumar R, Gruis N, Doorn R. Genome-wide characterization of 5-hydoxymethylcytosine in melanoma reveals major differences with nevus. Genes Chromosom Cancer. 2020;59:366-74. https://doi.org/10.1002/gcc.22837.

25. Ramsawhook A, Ruzov A, Coyle B. Wilms'Tumor protein 1 and enzymatic oxidation of 5-methylcytosine in brain tumors: potential perspectives. Front Cell Dev Biol. 2018;6:26

26. Lian CG, Xu Y, Ceol C, Wu F, Larson A, Dresser K, et al. Loss of 5-hydroxymethylcytosine is an epigenetic hallmark of melanoma. Cell. 2012;150:1135-46.

27. Liu H, Xu T, Cheng Y, Jin MH, Chang MY, Shu Q, et al. Altered 5-hydroxymethylcytosine landscape in primary gastric adenocarcinoma. DNA Cell Biol. 2019;38:1-10.

28. Uribe-Lewis S, Stark R, Carroll T, Dunning MJ, Bachman M, Ito Y, et al. 5-hydroxymethylcytosine marks promoters in colon that resist DNA hypermethylation in cancer. Genome Biol. 2015;16:1-15.

29. Chen K, Zhang J, Guo Z, Ma Q, Xu Z, Zhou Y, et al. Loss of 5-hydroxymethylcytosine is linked to gene body hypermethylation in kidney cancer. Cell Res. 2016;26:103-18.

30. Hu H, Shu M, He L, Yu X, Liu X, Lu Y, et al. Epigenomic landscape of 5-hydroxymethylcytosine reveals its transcriptional regulation of IncRNAs in colorectal cancer. Br J Cancer. 2017;116:658-68.

31. Kafer GR, Li X, Horii T, Suetake I, Tajima S, Hatada I, et al. 5-Hydroxymethylcytosine marks sites of DNA damage and promotes genome stability. Cell Rep. 2016;14:1283-92.

32. Mackay A, Burford A, Molinari V, Jones DTW, Izquierdo E, Brouwer-Visser J, et al. Molecular, pathological, radiological, and immune profiling of non-brainstem pediatric high-grade glioma from the HERBY phase II randomized trial. Cancer Cell. 2018;33:829-42.

33. Korshunov A, Ryzhova M, Hovestadt V, Bender S, Sturm D, Capper D, et al. Integrated analysis of pediatric glioblastoma reveals a subset of biologically favorable tumors with associated molecular prognostic markers. Acta Neuropathol. 2015;129:669-78.

34. Danielsson A, Nemes S, Tisell M, Lannering B, Nordborg C, Sabel M, et al. MethPed: a DNA methylation classifier tool for the identification of pediatric brain tumor subtypes. Clin Epigenet. 2015;7:1-9.

35. Ahamed MT, Danielsson A, Nemes S, Carén H. MethPed: an R package for the identification of pediatric brain tumor subtypes. BMC Bioinform. 2016;17:262. https://doi.org/10.1186/s12859-016-1144-0.

36. Capper D, Jones DTW, Sill M, Hovestadt V, Schrimpf D, Sturm D, et al. DNA methylation-based classification of central nervous system tumours. Nature. 2018;555:469-74. 
37. Hovestadt V, Jones DTW, Picelli S, Wang W, Kool M, Northcott PA, et al. Decoding the regulatory landscape of medulloblastoma using DNA methylation sequencing. Nature. 2014;510:537-41.

38. Noushmehr H, Weisenberger DJ, Diefes K, Phillips HS, Pujara K, Berman BP, et al. Identification of a $\mathrm{CpG}$ island methylator phenotype that defines a distinct subgroup of glioma. Cancer Cell. 2010;17:510-22. https://doi.org/ 10.1016/j.ccr.2010.03.017.

39. Houseman EA, Johnson KC, Christensen BC. OxyBS: estimation of 5-methylcytosine and 5-hydroxymethylcytosine from tandem-treated oxidative bisulfite and bisulfite DNA. Bioinformatics. 2016;32:2505-7.

40. Wu T, Zhang ZW, Li S, Wang B, Yang Z, Li P, et al. Characterization of global 5-hydroxymethylcytosine in pediatric posterior fossa ependymoma. Clin Epigenet. 2020;12:1-9.

41. Jin SG, Jiang Y, Qiu R, Rauch TA, Wang Y, Schackert G, et al. 5-hydroxymethylcytosine is strongly depleted in human cancers but its levels do not correlate with IDH1 mutations. Cancer Res. 2011;71:7360-5.

42. Heyn H, Vidal E, Ferreira HJ, Vizoso M, Sayols S, Gomez A, et al. Epigenomic analysis detects aberrant super-enhancer DNA methylation in human cancer. Genome Biol. 2016;17:11. https://doi.org/10.1186/ s13059-016-0879-2.

43. Khan A, Zhang X. dbSUPER: a database of super-enhancers in mouse and human genome. Nucleic Acids Res. 2016;44:D164-71.

44. Tsutsumi A, Kawamata T, Izumi N, Seitz H, Tomari Y. Recognition of the pre-miRNA structure by Drosophila-Dicer-1. Nat Struct Mol Biol. 2010;18:1153-8.

45. Jiang F, Ye X, Liu X, Fincher L, McKearin D, Liu Q. Dicer-1 and R3D1-L catalyze microRNA maturation in Drosophila. Genes Dev. 2005;19:1674-9.

46. Rand TA, Petersen $S$, Du F, Wang X. Argonaute2 cleaves the anti-guide strand of siRNA during RISC activation. Cell. 2005:123:621-9.

47. Shirley MD, Tang H, Gallione CJ, Baugher JD, Frelin LP, Cohen B, et al. Sturge-Weber syndrome and port-wine stains caused by somatic mutation in GNAQ. N Engl J Med. 2013;368:1971-9. https://doi.org/10.1056/ NEJMoa1213507.

48. Renault VM, Rafalski VA, Morgan AA, Salih DAM, Brett JO, Webb AE, et al. FoxO3 regulates neural stem cell homeostasis. Cell Stem. 2009;5:527-39.

49. Kurabayashi N, Nguyen MD, Sanada K. The G protein-coupled receptor GPRC5B contributes to neurogenesis in the developing mouse neocortex. Development. 2013;140:4335-46.

50. Marina RJ, Sturgill D, Bailly MA, Thenoz M, Varma G, Prigge MF, et al. TET-catalyzed oxidation of intragenic 5-methylcytosine regulates CTCFdependent alternative splicing. EMBO J. 2016;35:335-55. https://doi.org/ 10.15252/embj.201593235.

51. Houseman EA, Christensen BC, Yeh RF, Marsit CJ, Karagas MR, Wrensch M, et al. Model-based clustering of DNA methylation array data: a recursivepartitioning algorithm for high-dimensional data arising as a mixture of beta distributions. BMC Bioinform. 2008:9:1-15.

52. Qin Y, Feng $H$, Chen $M$, Wu H, Zheng X. InfiniumPurify: an R package for estimating and accounting for tumor purity in cancer methylation research. Genes Dis. 2018;5:43-5.

53. Wu G. Somatic histone H3 alterations in pediatric diffuse intrinsic pontine gliomas and non-brainstem glioblastomas. Nat Genet. 2012;44:251-3.

54. Lee RS, Stewart C, Carter SL, Ambrogio L, Cibulskis K, Sougnez C, et al. A remarkably simple genome underlies highly malignant pediatric rhabdoid cancers. J Clin Invest. 2012;122:2983-8.
55. Mack SC, Witt H, Piro RM, Gu L, Zuyderduyn S, Stütz AM, et al. Epigenomic alterations define lethal CIMP-positive ependymomas of infancy. Nature. 2014:506:445-50.

56. Kraus TFJ, Globisch D, Wagner M, Eigenbrod S, Widmann D, Münzel M, et al. Low values of 5 -hydroxymethylcytosine $(5 \mathrm{hmC})$, the "sixth base" are associated with anaplasia in human brain tumors. Int $\mathrm{J}$ Cancer. 2012;131:1577-90. https://doi.org/10.1002/ijc.27429.

57. Baker SJ, Ellison DW, Gutmann DH. Pediatric gliomas as neurodevelopmental disorders. Glia. 2015. https://doi.org/10.1002/glia.22945.

58. Wen L, Li X, Yan L, Tan Y, Li R, Zhao Y, et al. Whole-genome analysis of 5-hydroxymethylcytosine and 5-methylcytosine at base resolution in the human brain. Genome Biol. 2014;15:R49. https://doi.org/10.1186/ gb-2014-15-3-r49.

59. Gross JA, Pacis A, Chen GG, Barreiro LB, Ernst C, Turecki G. Characterizing 5-hydroxymethylcytosine in human prefrontal cortex at single base resolution. BMC Genomics. 2015;16:1-14.

60. Teif VB, Beshnova DA, Vainshtein Y, Marth C, Mallm JP, Rippe TH. Nucleosome repositioning links DNA (de)methylation and differential CTCF binding during stem cell development. Genome Res. 2014;24:1285-95.

61. Sun Z, Terragni J, Borgaro JG, Liu Y, Yu L, Guan S, et al. High-resolution enzymatic mapping of genomic 5-hydroxymethylcytosine in mouse embryonic stem cells. Cell Rep. 2013;3:567-76.

62. Mahajan V, Farquhar C, Ponnampalam AP. Could DNA hydroxymethylation be crucial in influencing steroid hormone signaling in endometrial biology and endometriosis? Mol Reprod Dev. 2020;87:7-16.

63. Zhao J, Ma X, Ma J, Sun L, Lu B, Wang Y, et al. TET3 mediates alterations in the epigenetic marker $5 \mathrm{hmC}$ and Akt pathway in steroid-associated osteonecrosis. J Bone Miner Res. 2017;32:319-32. https://doi.org/10.1002/ jbmr.2992.

64. Fortin J-P, Triche TJ, Hansen KD. Preprocessing, normalization and integration of the illumina humanmethylation EPIC array with minfi. Bioinformatics. 2016. https://doi.org/10.1093/bioinformatics/btw691.

65. Zhou W, Laird PW, Shen H. Comprehensive characterization, annotation and innovative use of Infinium DNA methylation BeadChip probes. Nucleic Acids Res. 2017:45:e22.

66. R Core Team. R: a language and environment for statistical computing. Vienna: R Foundation for Statistical Computing; 2019.

67. Ritchie ME, Phipson B, Wu D, Hu Y, Law CW, Shi W, et al. limma powers differential expression analyses for RNA-sequencing and microarray studies. Nucleic Acids Res. 2015;43:e47.

68. McLean CY, Bristor D, Hiller M, Clarke SL, Schaar BT, Lowe CB, et al. GREAT improves functional interpretation of cis-regulatory regions. Nat Biotechnol. 2010;28:495-501.

69. Sheffield NC, Bock C. LOLA: enrichment analysis for genomic region sets and regulatory elements in $\mathrm{R}$ and Bioconductor. Bioinformatics. 2015;32:587-9.

\section{Publisher's Note}

Springer Nature remains neutral with regard to jurisdictional claims in published maps and institutional affiliations.

Ready to submit your research? Choose BMC and benefit from:

- fast, convenient online submission

- thorough peer review by experienced researchers in your field

- rapid publication on acceptance

- support for research data, including large and complex data types

- gold Open Access which fosters wider collaboration and increased citations

- maximum visibility for your research: over 100M website views per year

At BMC, research is always in progress.

Learn more biomedcentral.com/submissions 